\title{
Microstructures of cellulose coagulated in water and alcohols from 1-ethyl-3-methylimidazolium acetate: contrasting coagulation mechanisms
}

\author{
Artur Hedlund (D) Tobias Köhnke • Joel Hagman • Ulf Olsson • \\ Hans Theliander
}

Received: 23 March 2018/Accepted: 30 November 2018/Published online: 15 December 2018

(C) The Author(s) 2018

\begin{abstract}
Coagulation of cellulose solutions is a process whereby many useful materials with variable microstructures and properties can be produced. This study investigates the complexity of the phase separation that generates the structural heterogeneity of such materials. The ionic liquid, 1-ethyl-3-methylimidazolium acetate $\left(\left[\mathrm{C}_{2} \mathrm{mim}\right][\mathrm{OAc}]\right)$, and a co-solvent, dimethylsulfoxide (DMSO), are used to dissolve microcrystalline cellulose in concentrations from 5 to $25 \mathrm{wt} \%$. The solutions are coagulated in water or 2-propanol (2PrOH). The coagulated material is then washed and solvent exchanged (water $\rightarrow 2 \mathrm{PrOH} \rightarrow$ butanone $\rightarrow$ cyclohexane) in order to preserve the generated microstructures upon
\end{abstract}

subsequent drying before analysis. Sweep electron microscopy images of $50 \mathrm{k}$ magnification reveal openpore fibrillar structures. The crystalline constituents of those fibrils are estimated using wide-angle X-ray spectroscopy and specific surface area data. It is found that the crystalline order or crystallite size is reduced by an increase in cellulose concentration, by the use of the co-solvent DMSO, or by the use of $2 \mathrm{PrOH}$ instead of water as the coagulant. Because previous theories cannot explain these trends, an alternative explanation is presented here focused on solid-liquid versus liquid-liquid phase separations.

A. Hedlund $(\bowtie) \cdot$ T. Köhnke

Biobased Fibers, Swerea IVF, Argongatan 30,

43153 Mölndal, Sweden

e-mail: artur.hedlund@ri.se

A. Hedlund $\cdot$ H. Theliander

Forest Products and Chemical Engineering, Chalmers

University of Technology, Gothenburg, Sweden

J. Hagman · U. Olsson

Physical Chemistry, Lund University, Box 124,

22100 Lund, Sweden

H. Theliander

Wallenberg Wood Science Center, The Royal Institute of

Technology, Chalmers University of Technology,

10044 Stockholm, Sweden 


\section{Graphical abstract}

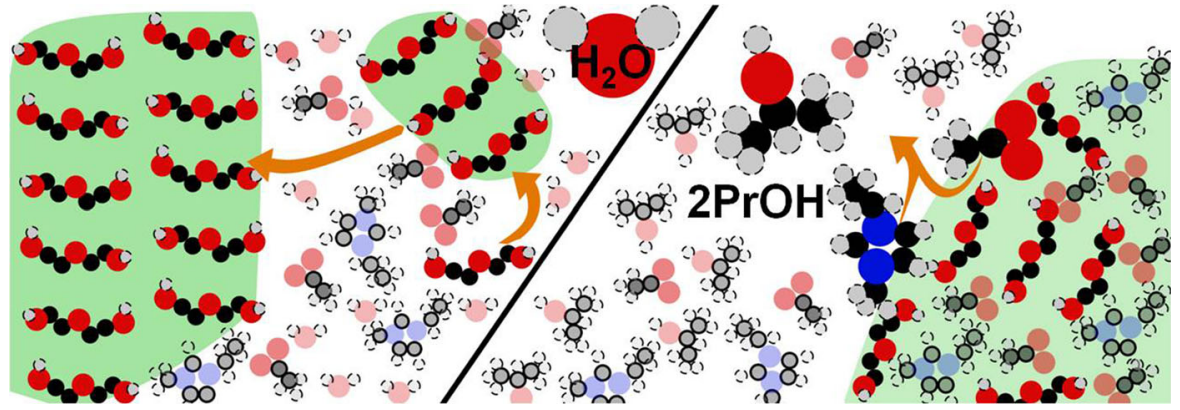

Keywords Cellulose - Ionic liquid · Microstructure · Regeneration $\cdot$ Co-solvent $\cdot$ Non-solvent

\section{Introduction}

Cellulose is the major biopolymer that provides mechanical reinforcement of the cell walls in landliving plants. In most native supramolecular structures of cellulose, the polymers are hierarchically organized in crystalline nanofibrils, which are bundled into larger fibrils that are wound at an angle around the plant cell wall. There are numerous applications in which cellulose is used in its native fibrillar structure, such as cotton, lumber, fiber boards, paper and many more. For cellulose to be used as material in other than its native form, shaping by dissolution and precipitation is necessary. The most important example of such a process is the viscose process, which has been used for more than 100 years on the industrial scale. Even though several other solvent systems for cellulose are available, NMMO is still, decades after its industrialization, the only new cellulose solvent in industrial use. However, at $0.2 \mathrm{Mton} / \mathrm{a}$ of fiber, lyocell remains a niche-product relative to the $5 \mathrm{Mton} / \mathrm{a}$ of viscose (The_Fiber_Year_GmbH 2017). This is probably due to issues with process safety and related costs. Therefore, the toxic viscose process continues to increase in volume. In order to expand the use of cellulosic materials, new sustainable and resourceefficient cellulose dissolution-regeneration processes are required together with the ability to control material properties. To succeed in this endeavor, the effects of process conditions on the properties of the precipitated cellulosic material from new solvents must be better understood.

Ionic liquids (ILs) are bulky organic salts that are liquids at temperatures below $100{ }^{\circ} \mathrm{C}$. Several ILs are among the most recently added to the list of available cellulose solvents (Hermanutz et al. 2008; Swatloski et al. 2002). The properties of IL-cellulose solutions have been intensely studied over the last decades. Concentrated solutions of more than $25 \mathrm{wt} \%$ cellulose can be obtained, e.g. in 1-ethyl-3-methylimidazolium acetate $\left[\mathrm{C}_{2} \mathrm{mim}\right][\mathrm{OAc}]$. The viscosities of concentrated IL-cellulose solutions can be very high, which poses problems for processing. Consequently, several aspects of their rheology have been studied intensively: shear rheology (Collier et al. 2009; Sammons et al. 2008a), elongational viscosity (Sammons et al. 2008b), mesophases and anisotropic solutions (Kosan et al. 2010; Song et al. 2011), and intrinsic viscosity (Le et al. 2012). The addition of certain co-solvents can reduce the viscosity as well as the consumption of IL. DMSO and certain other polar aprotic compounds have shown the greatest potential for this purpose. Several aspects of the use of these compounds in mixtures with ILs have been studied: cellulose solubility (Andanson et al. 2014; Hedlund et al. 2015; Idström et al. 2017; Le et al. 2014; Olsson et al. 2014), fiber spinning (Härdelin et al. 2012; Olsson et al. 2014; Xu et al. 2008), viscosity (Lu et al. 2015; Saba et al. 2014), and self-diffusion (Radhi et al. 2015). Although the use of co-solvents enables solvent properties to be tuned, their effect on precipitated cellulose structures and properties have not been reported.

Another aspect of ILs that has recently been clarified is the importance of anion basicity for the dissolution of cellulose. The aspects of solvent 
basicity and acidity have been studied and quantified with Kamlet-Taft parameters (Hauru et al. 2012; Parviainen et al. 2013). Generally, a high basicity is required to disrupt cellulose-cellulose hydrogen bonds. The IL anions form thermodynamically more favorable hydrogen bonds with cellulose hydroxyl groups. (Zhang et al. 2010) The process opposite of dissolution is precipitation, and it is induced by coagulants, which are typically water or other simple protic solvents like alcohols. The main function of the coagulant is to substitute for cellulose hydroxyls in the interaction with IL anions. Cellulose-cellulose hydrogen bonds are reformed and aggregation starts as a consequence of IL-anion removal from cellulose hydroxyl groups (Gupta et al. 2013a, b). Similar to dissolution, solvent quality during precipitation may also be quantified using Kamlet-Taft parameters, which shift to lower basicities as more coagulant is added (Hauru et al. 2012).

Most studies of cellulose structures precipitated from ILs have been performed with very specific applications in mind, cf. a recent review (Wang et al. 2016). For this reason, a more general understanding of the effect of certain coagulation conditions on formed structures is lacking. It has been found, for example, that $\left[\mathrm{C}_{2} \mathrm{mim}\right][\mathrm{OAc}]$-cellulose solutions produce transparent films if coagulated in ethanol, as opposed to opaque films if coagulated in water (Wawro et al. 2014). Fiber spinning experiments have been performed using several different ILs, with airgap (Kosan et al. 2008; Michud et al. 2015, 2016) and conventional wet-spinning (Olsson and Westman 2013). Most of these investigations have focused on the characterization of mechanical fiber properties, whereas the few microscopic properties tested have been specific to fibers and their molecular orientation, such as birefringence and crystalline orientation. Crystalline index (CI) measurements (using WAXS or CP/MAS NMR) and SEM images of fiber cross sections have also been frequent sources of information in these studies. Evidently much detail is still lacking in understanding the kind of structures that are formed under given process conditions. However, some conclusions from the investigations of structures formed from NMMO-based cellulose solutions may also be applicable to ILs, thus reducing the knowledge deficit in this field to some extent. One study (Bang et al. 1999) has shown that an increase in cellulose $M_{w}$ and concentration, as well as a decrease in NMMO concentration in the coagulation liquid, induce a modest decline in CI and crystallite size in films coagulated in water. All three investigated parameters affect either the mobility of cellulose chains or the time available for cellulose chains to organize during coagulation. Consequently, that study concluded that mobility was the main parameter correlating to crystallinity and crystallite size.

The aim of this study is to better understand the formation of nanostructures during the precipitation of cellulose from ILs. To do so, a set of different concentrations from 5 to $25 \mathrm{wt} \%$ of microcrystalline cellulose (MCC) dissolved in $\left[\mathrm{C}_{2} \mathrm{mim}\right][\mathrm{OAc}]$ were coagulated in either deionized water or isopropanol $(2 \mathrm{PrOH})$. After coagulation and washing in the coagulant, solvent exchange was performed through a series of decreasingly polar solvents. The gently dried solvent-exchanged material was then characterized using BET measurements of specific surface area (SSA), wide angle X-ray scattering (WAXS), and SEM imaging. The structural changes usually induced by drying could be avoided using this procedure, and structures originally coagulated could be studied.

\section{Materials and methods}

\section{Materials}

$\left[\mathrm{C}_{2} \mathrm{mim}\right][\mathrm{OAc}]$ of purity $>90 \%$, produced by BASF, was purchased from Sigma Aldrich. The moisture content was determined to be $0.5 \%$ using Karl-Fischer titration. Anhydrous DMSO with molecular sieve was also purchased from Sigma Aldrich. The cellulose used was microcrystalline cellulose (MCC) PH-101 with $\mathrm{M}_{\mathrm{w}}=28.4 \mathrm{kDa}, \mathrm{M}_{\mathrm{w}} / \mathrm{M}_{\mathrm{n}}=2.6$. De-ionized water and $2 \mathrm{PrOH}(>99.5$ wt \%) were used as coagulants.

Table 1 Solution designations explained with their respective compositions

\begin{tabular}{lllr}
\hline Solution designation & $\begin{array}{l}\mathrm{wt} \% \\
\mathrm{MCC}\end{array}$ & $\begin{array}{l}\mathrm{wt} \% \\
{\left[\mathrm{C}_{2} \text { mim] }\right.}\end{array}[\mathrm{OAc}]$ & $\begin{array}{r}\mathrm{wt} \% \\
\mathrm{DMSO}\end{array}$ \\
\hline 99:1-5\%MCC & 5 & 94.05 & 0.95 \\
99:1-14.3\%MCC & 14.3 & 84.85 & 0.85 \\
99:1-25\%MCC & 25 & 74.25 & 0.75 \\
50:50-14.3\%MCC & 14.3 & 42.85 & 42.85 \\
\hline
\end{tabular}


Solution preparation

The solvents used were mixtures of $\left[\mathrm{C}_{2} \mathrm{mim}\right][\mathrm{OAc}]$ and DMSO of either 99:1 or 50:50 composition $\left(\mathrm{wt}_{[\mathrm{C} 2 \mathrm{mim}][\mathrm{OAc}]}: \mathrm{wt}_{\mathrm{DMSO}}\right)$. The objective of the addition of $1 \mathrm{wt} \%$ of DMSO to the "pure" $\left[\mathrm{C}_{2} \mathrm{mim}\right][\mathrm{OAc}]$ solvent was to use it as a tracer in a parallel study on mass transport (Hedlund et al. 2017), The set of four different solutions prepared for the experiments are summarized in Table 1. MCC was added to the solvent at room temperature and stirred with an overhead mixer. The stirring speed was set at 800 RPM initially and, then, lowered to a level that did not cause the solution temperature to rise above $80{ }^{\circ} \mathrm{C}$. The whole contraption was arranged so that no moisture could enter during stirring, which continued for $2 \mathrm{~h}$. The solutions were confirmed to be fully dissolved by verifying the absence of undissolved material between cross polarizing plates under a microscope. Air trapped during stirring was removed from the solution with centrifugation before the solution was transferred to a syringe for moisture-free storage until use (within a week at room temperature).

\section{Coagulation}

Coagulation was performed by covering a steel rod (diameter of $4 \mathrm{~mm}$ ) with a $0.35 \mathrm{~mm}$ thick solution layer and immersing the rod in water or $2 \mathrm{PrOH}$ for a sufficient amount of time to coagulate the full layer thickness, cf. (Hedlund et al. 2017). The resulting cellulose tube ( $\sim 0.3 \mathrm{~g}$ wet) was pulled off the rod and stored in a small flask with about $4 \mathrm{ml}$ of the coagulant used.

\section{Solvent exchange}

In order to maintain the coagulated structure during drying, the coagulant was solvent exchanged to cyclohexane using the following sequence: water to $2 \mathrm{PrOH}$, to butanone, and then to cyclohexane with three exchanges of liquid per solvent. The liquid exchanged in each cycle was in excess of 15 times the sample mass. The material coagulated in alcohols was treated using two different methods: one involving a water washing step and the other starting from $2 \mathrm{PrOH}$, which excluded water from the sequence.

\section{BET}

The solvent-exchanged samples were introduced into the BET measurement glass tubes, still soaked in cyclohexane, which was removed with a stream of dry $\mathrm{N}_{2}$ at room temperature for more than $10 \mathrm{~h}$ prior to starting the measurements. The measurements were performed on a Micrometrics TriStar 3000 Surface Area and Porosity Analyzer using a standard routine with 10 measurement points for adsorption and desorption in the pressure range of $0.01-0.15$ atmospheres. The Brunauer-Emmett-Teller (BET) equation (Brunauer et al. 1938) was applied to the $\mathrm{N}_{2}$ adsorption isotherms at $77 \mathrm{~K}$, to determine the specific surface areas.

\section{SEM}

The material, solvent exchanged and dried as previously described, was torn at room temperature so as to expose its fractured cross section, and then covered with $4 \mathrm{~nm}$ of Pt. This coverage was estimated based on its exposure time in the sputtering device. The actual coverage of nanostructures, given their large porosity, must be significantly less. Images of specimen cross sections were then taken with a Scanning Electron Microscope (SEM) from JEOL, model JSM-7800F. Secondary Electron (SE) images of $50 \mathrm{k}$ magnification were acquired using an accelerating voltage of $3 \mathrm{kV}$ and a working distance of 6-7 $\mathrm{mm}$.

X-ray scattering

Wide-angle X-ray scattering measurements were performed using a SAXSLab Ganesha 300XL instrument (SAXSLAB ApS, Skovlunde, Denmark). It is a pinhole collimated system equipped with a Genix 3D X-ray source (Xenocs SA, Sassenage, France). Data were collected with the detector placed at various sample-to-detector positions, which yielded an overall $q$ range of $7.5 \times 10^{-2}$ to $2.6 \AA^{-1}$. The dry samples were fixed perpendicular to the beam on a sample stage for single-use capillaries. The two-dimensional (2D) scattering pattern was recorded using a 2D $300 \mathrm{k}$ Pilatus detector (Dectris Ltd., Baden, Switzerland) and radially averaged using SAXSGui software to obtain the scattered intensity $I(q)$, where $q=$ $(4 \pi / \lambda) \sin (\theta / 2)$ was the scattering vector, $\theta$ was the 
scattering angle, and $\lambda=1.54 \AA$ was the X-ray wavelength. Exposure times were $60 \mathrm{~s}$.

The data was analyzed by deconvoluting the data $I(q)$ over the $q$-range of $0.7-1.75 \AA^{-1}$ into the three well-known crystalline peaks $0.87,1.41$, and $1.56 \AA^{-1}$ (corresponding to $2 \theta$ of $12.2^{\circ}, 19.9^{\circ}$, and $22^{\circ}$ ) (French 2014; French and Cintrón 2013) and an amorphous broad peak with a maximum around $q$ equal to $1.2 \AA^{-1}$ $\left(2 \theta=17^{\circ}\right)$. The crystalline peaks were represented by Cauchy (Lorentzian) functions, Eq. 1, (Gjönnes et al. 1958).

$L(q)=K \frac{\alpha}{\alpha^{2}+\left(q-q_{0}\right)^{2}}$

$\alpha$ is half the "peak width at half maximum" (pwhm), $q_{0}$ is the peak position, and $\mathrm{K}$ is the surface of its integral. The shape of the amorphous peak is not well known, and here it was modeled using $L(q) * q$. The reason for using this function was its relatively general correlation with the different spectra measured. Their amorphous parts were quite asymmetrical and, therefore, could not be satisfactorily modeled using symmetrical functions, such as Cauchy- or Gauss curves, without the additional asymmetrical factor of $q$. There are many alternative functions that could be used for this purpose, however, an important requirement of any such function is that it is relatively flat in the range of the main crystalline peak at $1.41 \AA^{-1}\left(2 \theta=19.9^{\circ}\right)$. This is to maintain the evaluated width of the peak at $1.41 \AA^{-1}$ relatively independent of the exact position of the amorphous peak. Least-squares fitting was applied to calculate the peak heights, $K / \alpha$, and an additional computerized optimization function was applied to find the optimal peak width parameter, $\alpha$. With these tools (Matlab scripts), the parameters of the peaks' central positions, $q_{0}$, were adapted manually, as they were not found at the exact positions previously reported. The main output of this procedure was the pwhm of the main peak at $1.41 \AA^{-1}$ and, in some cases, also the pwhm of the smaller peak at $0.87 \AA^{-1}$ $\left(2 \theta=12.2^{\circ}\right)$. The crystallite lateral dimensions were then obtained using the Scherrer equation, Eq. 2 (where the pwhm is expressed in $q$, as opposed to $s=q /(2 \pi)$ or $2 \theta)$.

$D=k \frac{2 \pi * \lambda}{p w h m * \cos \theta}$ $k$ is the "form factor," which can often be approximated to 1 , given that the precision is generally limited by other factors. There can be additional factors behind line broadening, such as the width of the beam and paracrystalline defects, which should be withdrawn from the observed pwhm to obtain the true pwhm (Balta-Calleja and Vonk 1989). However, it is generally accepted that limited crystallite size is the main factor behind the broadened crystalline reflections observed (French 2014; French and Cintrón 2013). In randomly oriented cellulose-II materials, there is also a small reflection (012) at $2 \theta=20.5$ that can make the peak at $2 \theta=19.9^{\circ},(110)$, appear broader (French 2014). However, given the already large pwhm of (110) and small height of (012) relative to (110), the effect of the overlapping of (110) and (012) on the pwhm of (110) should be marginal relative to the total pwhm. For the purpose of this article, i.e. to obtain comparability within a set of relatively similar samples and an approximate absolute size, it was, therefore, considered sufficient to use the observed pwhm.

\section{Results and discussion}

The set of solutions, presented in Table 1, were coagulated in water or $2 \mathrm{PrOH}$ and, thereafter, solventexchanged and dried under dry $\mathrm{N}_{2}$, at room temperature. Here follows an account of the results and an attempt to interpret the structural characteristics of the resulting materials in terms of the phase-separation processes and conditions in which they were created.

As an introductory note, it should be stated that, in most immersion precipitation processes, phase separation occurs by liquid-liquid phase separation. However, for polymers that have a strong tendency to crystallize, like cellulose, a solid-liquid mode of separation may be thermodynamically favorable. In some cases, these two modes of separation are in competition and can even combine in sequence. In such a case, the solid-liquid mode is generally more thermodynamically favorable but can still be dynamically unfavorable due to the slow dynamics of crystallization, which can result in the prevalence of the liquid-liquid separation mode. The liquid-liquid separation can then be followed by solid-liquid separation in the polymer rich phase. Generally, this 

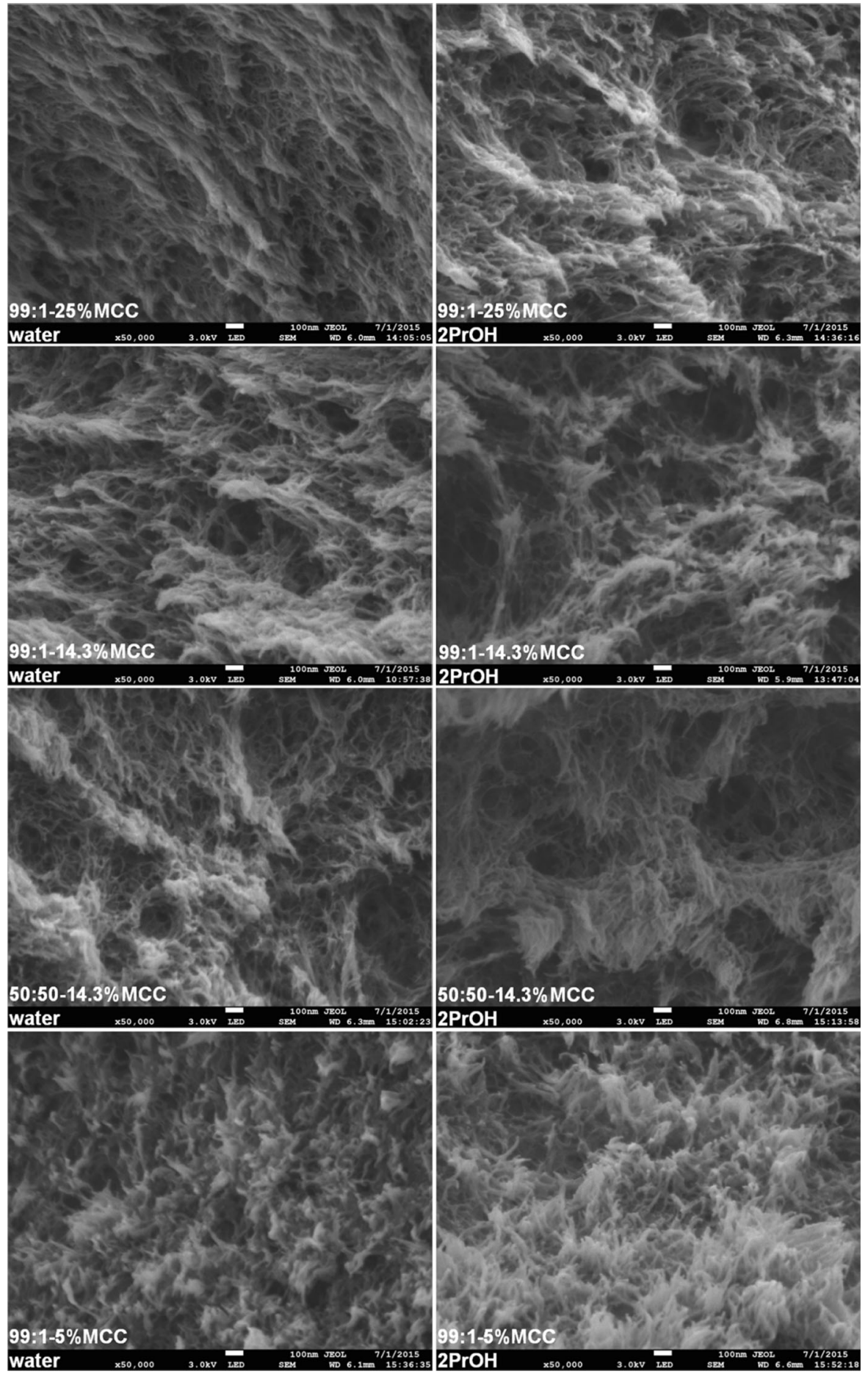

Fig. $150 \mathrm{k}$ Magnification SEM images of the nanostructure of coagulated and solvent-exchanged dry material coagulated in water (left) or in $2 \mathrm{PrOH}$ (right) 


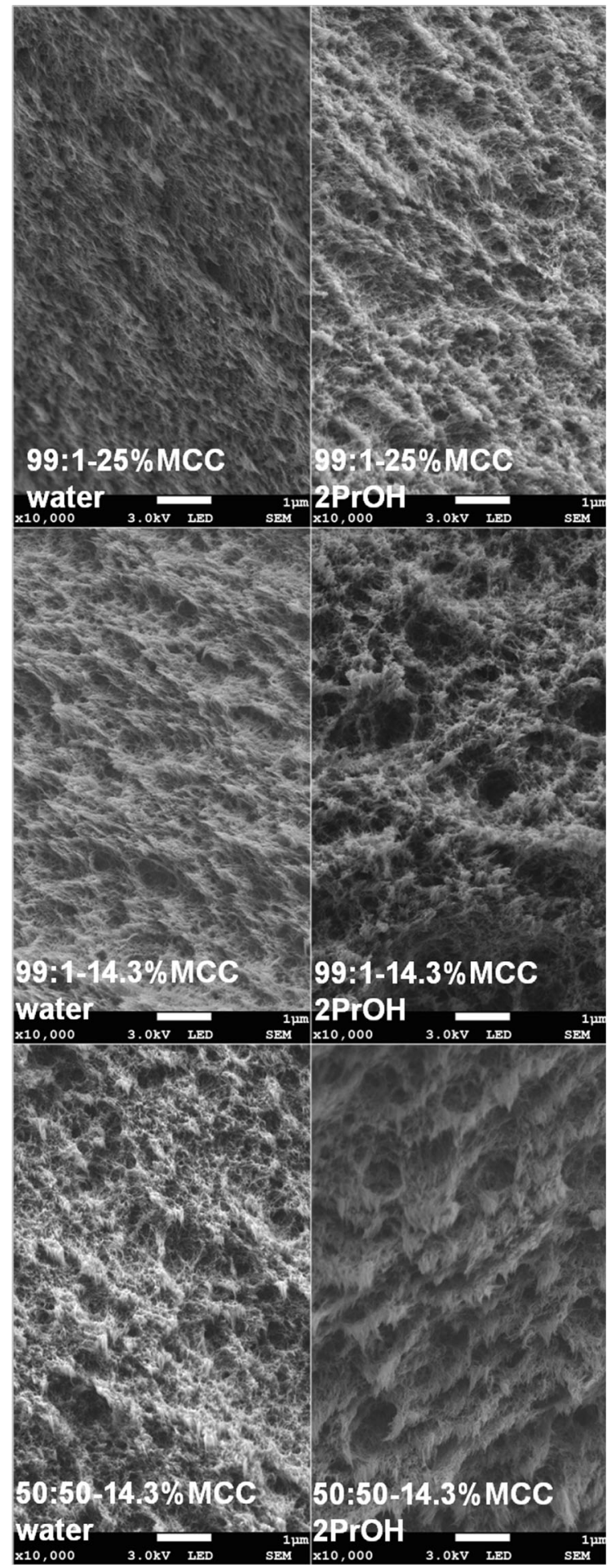

Fig. $210 \mathrm{k}$ Times magnification SEM images for overview of coagulated and solvent-exchanged dry material coagulated in water (left) or in $2 \mathrm{PrOH}$ (right) secondary solid liquid separation transforms the polymer-rich phase either into a dense phase by vitrification or into an open gel network of interconnected crystallites (Burghardt et al. 1987; Van de Witte et al. 1996). Before returning to the issue of liquid-liquid versus solid-liquid separations, in the latter part of this section, we will apply the most standard assumption about the mode of phase separation; separation occurs by the liquid-liquid mode and the cellulose concentration of the cellulose-rich phase, as initially separated, is close to the solubility limit in $\left[\mathrm{C}_{2} \mathrm{mim}\right][\mathrm{OAc}]$ of $\sim 30 \mathrm{wt} \%$ (Kosan et al. 2010). This would imply that the respective amounts of each phase should be rather similar for the 99:1-14.3\% MCC and 50:50-14.3\% MCC solutions. Whereas the cellulose poor phase and rich phase should dominate in the 99:1-5\% MCC and 99:1-25\% MCC solutions, respectively. The weight fractions of the major and minor phases would in these cases be in a relation of $\sim 5: 1$.

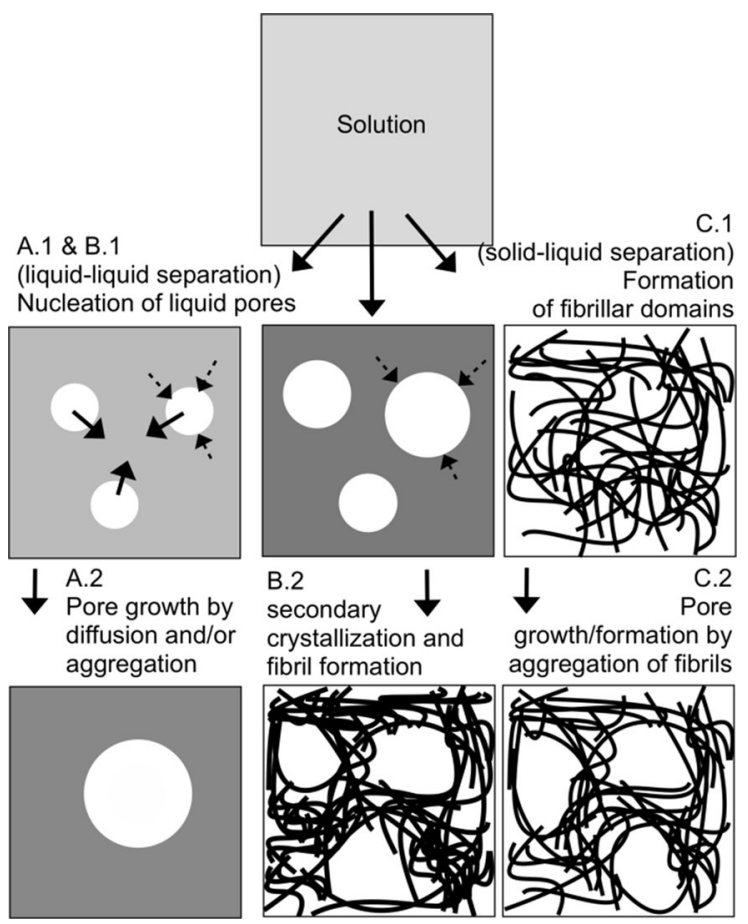

Fig. 3 Formation and growth of pores by various routes: a secondary processes of pore growth, after liquid-liquid phase separation, Ostwald-ripening and aggregation; $\mathbf{b}$ formation of the nano-porosity surrounding larger pores that formed during liquid-liquid separation,prior to the final phase separation; c secondary pore formation by fibril movements after primary solid-liquid separation 


\section{SEM images}

The fracture surfaces of coagulated material show open-pore fibrillar structures, Fig. 1. Bicontinuous structures like these, studied, e.g. within the field of membrane science, are generally attributed to either binodal decomposition or to spinodal decomposition. The regular periodicity of the finest structures in these networks is an indication that the final separation step of crystallite and fibril formation occurs by spinodal decomposition. However, this commonly discussed issue will not be elaborated in this study, because the results point mainly to important differences prior to the final formation of fibrils.

Figures 1 and 2 show variations in fibril aggregation. The frequency and size of individual larger pores in-between such larger fibril structures are the most apparent differences between the different microstructures. With $2 \mathrm{PrOH}$ as the coagulant, more large pores, i.e. more heterogeneous structures, are produced than in water. Based on comparisons of the SEM images of different samples, it is apparent that the material that was coagulated from the $5 \mathrm{wt} \%$ cellulose solution is denser than expected. A previous study (Hedlund et al. 2017) found that the dry content of the cellulose structure before solvent exchange and drying correlates with each solution's cellulose concentration. This indicates that the $5 \mathrm{wt} \%$ cellulose material underwent a large degree of volume collapse during solvent exchange and drying, which disqualifies it from the general comparison of pore structures observed using SEM. A comparison between the materials coagulated from $14.3 \mathrm{wt} \%$ - and $25 \mathrm{wt} \%$ cellulose solutions shows that the higher concentration generates a denser, more even material, with fewer large pores. In contrast, increasing the DMSO content has the effect of increasing the size of individual larger pores.

Figure 3 shows a summary of the plausible origins of individual larger pores. There are two generally accepted mechanisms for the growth of pores: Ostwald ripening and the aggregation of smaller liquid phase domains, illustrated by route A in Fig. 3 (Burghardt et al. 1987; Van de Witte et al. 1996). The very high viscosity of the cellulose-rich phase reduces the likelihood of motion of isolated non-solvent-rich domains. However, the bicontinuous morphology that appears to be the present case would be permeable to the convection of the non-solvent phase as long as the network of the cellulose phase is sufficiently flexible

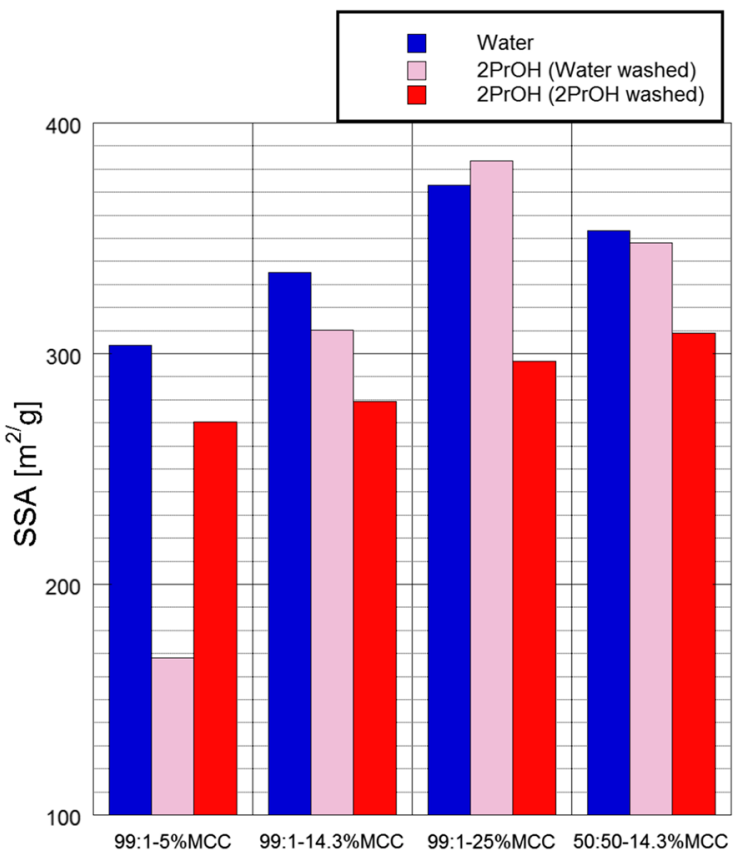

Fig. 4 SSA values measured using BET $\mathrm{N}_{2}$-adsorption for material coagulated in water or $2 \mathrm{PrOH}$. The latter set of samples show significant differences depending on the washing procedure applied (with or without water) prior to solvent exchange

to accommodate the incoming volume. Thus, it is conceivable that once crystallites or fibrils of the cellulose-rich phase have formed, the ones in close proximity to each other, could move a short distance in order to touch and reduce their interface to the surrounding non-solvent, as illustrated by route $\mathrm{C}$ in Fig. 3. This is a likely origin of small pores, such as the pores developed in water, without DMSO. However, it is an improbable explanation for the individual larger pores seen in some materials, such as those formed in $2 \mathrm{PrOH}$ and, in particular, those formed from 50:50 to $14.3 \%$ MCC solutions, Fig. 1. It is not likely that solid or semi-solid fibrils could move the rather long distances required to form these pores.

A more probable explanation for the individual larger pores is the primary separation of liquid nonsolvent-rich droplets, which increase in size due to diffusion from the surrounding phase, as schematically described in Fig. 3, route B. The larger pores that are formed with DMSO in the solvent could be an effect of enhanced diffusion through the cellulose-rich phase with DMSO present; faster diffusion would produce larger pores before the growth of pores is stopped by secondary phase separation. Then, during 


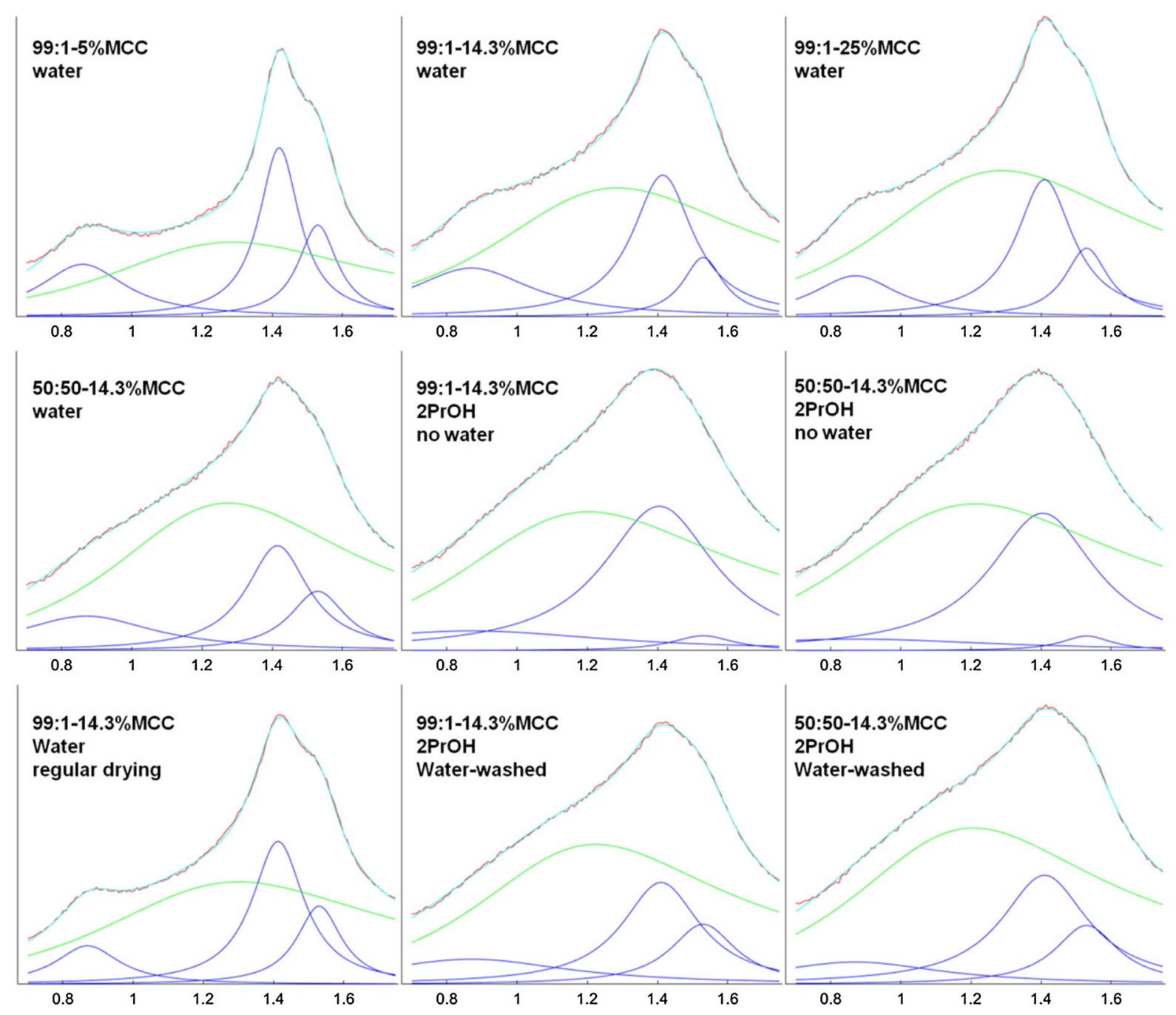

Fig. 5 WAXS spectra with their respective deconvolutions: amorphous, green; crystalline, blue; model sum, cyan; data, red (largely overlapping with the model sum, cyan). q-value in $\AA^{-1}$ on the $\mathrm{x}$-axis, linear intensity scale on the y-axis. (Color figure online)

that phase separation, the cellulose-rich phase itself would separate to form the nano-porous fibrillar structures that surround the larger pores already present. The lack of continuous compact structures in this material, as well as in the material coagulated in water, excludes vitrification as the process by which solid-liquid separation proceeds. Thus, in all cases, crystallization and the formation of interconnected fibrils into an open gel network is left as the most likely process.
Specific surface areas

The specific surface areas (SSA) may be translated into a measure of fibrillar diameter, $d$, assuming a round cross section, a large aspect ratio, and a monodisperse size distribution, by the equation:

$d=4 /(\rho * S S A)$

where $\rho$ is the density of the fibril $(\approx 1.55 \mathrm{~g} / \mathrm{ml})$. All but one of the materials have mean fibril diameters within the range of $8-10 \mathrm{~nm}$, according to Eq. 3. The assumption of fibrils of such diameters appears to be largely consistent with the SEM images. The SSAs, measured using BET and displayed in Fig. 4, show a 
Table 2 Summary of the parameters for the three crystalline peaks and the amorphous scattering found for the deconvolutions in Fig. 5

\begin{tabular}{|c|c|c|c|c|}
\hline Crystalline peak $(2 \theta)$ & $2 \theta \approx 12^{\circ}(1 \overline{1} 0)$ & $2 \theta \approx 20^{\circ}(110)$ & $2 \theta \approx 22^{\circ}(020)$ & Amorphous peak \\
\hline \multicolumn{5}{|l|}{$99: 1-5 \% \mathrm{MCC}$ water } \\
\hline $\mathrm{Q}_{0}\left(\AA^{-1}\right)$ & 0.860 & 1.420 & 1.530 & 1.200 \\
\hline $2 \theta$ & 12.1 & 20.0 & 21.6 & 16.9 \\
\hline$\alpha$ & 0.150 & 0.070 & 0.068 & 0.460 \\
\hline $\mathrm{K} / \alpha$ & 0.874 & 2.813 & 1.527 & 1.000 \\
\hline $\mathrm{D}(\AA)$ & 32 & 70 & 73 & 11 \\
\hline \multicolumn{5}{|l|}{$99: 1-14.3 \% \mathrm{MCC}$ water } \\
\hline $\mathrm{Q}_{0}\left(\AA^{-1}\right)$ & 0.870 & 1.415 & 1.530 & 1.200 \\
\hline $2 \theta$ & 12.2 & 20.0 & 21.6 & 16.9 \\
\hline$\alpha$ & 0.210 & 0.105 & 0.070 & 0.460 \\
\hline $\mathrm{K} / \alpha$ & 0.470 & 1.366 & 0.573 & 1.000 \\
\hline $\mathrm{D}(\AA)$ & 23 & 47 & 70 & 11 \\
\hline \multicolumn{5}{|c|}{$99: 1-14.3 \% \mathrm{MCC}$ water (regular drying) } \\
\hline $\mathrm{Q}_{0}\left(\AA^{-1}\right)$ & 0.870 & 1.413 & 1.530 & 1.200 \\
\hline $2 \theta$ & 12.2 & 19.9 & 21.6 & 16.9 \\
\hline$\alpha$ & 0.110 & 0.090 & 0.078 & 0.500 \\
\hline $\mathrm{K} / \alpha$ & 0.471 & 1.744 & 0.954 & 1.000 \\
\hline $\mathrm{D}(\AA)$ & 44 & 55 & 64 & 10 \\
\hline \multicolumn{5}{|c|}{$50: 50-14.3 \% \mathrm{MCC}$ water } \\
\hline $\mathrm{Q}_{0}\left(\AA^{-1}\right)$ & 0.870 & 1.415 & 1.530 & 1.200 \\
\hline $2 \theta$ & 12.2 & 20.0 & 21.6 & 16.9 \\
\hline$\alpha$ & 0.235 & 0.108 & 0.098 & 0.430 \\
\hline $\mathrm{K} / \alpha$ & 0.289 & 0.879 & 0.497 & 1.000 \\
\hline $\mathrm{D}(\AA)$ & 21 & 46 & 51 & 11 \\
\hline \multicolumn{5}{|l|}{ 99:1-25\% MCC water } \\
\hline $\mathrm{Q}_{0}\left(\AA^{-1}\right)$ & 0.870 & 1.410 & 1.530 & 1.200 \\
\hline $2 \theta$ & 12.2 & 19.9 & 21.6 & 16.9 \\
\hline$\alpha$ & 0.148 & 0.098 & 0.075 & 0.468 \\
\hline $\mathrm{K} / \alpha$ & 0.350 & 1.167 & 0.584 & 1.000 \\
\hline $\mathrm{D}(\AA)$ & 33 & 50 & 66 & 10 \\
\hline \multicolumn{5}{|c|}{ 99:1-14.3\% MCC 2PrOH (water-washed) } \\
\hline $\mathrm{Q}_{0}\left(\AA^{-1}\right)$ & 0.870 & 1.410 & 1.530 & 1.140 \\
\hline $2 \theta$ & 12.2 & 19.9 & 21.6 & 16.1 \\
\hline$\alpha$ & 0.338 & 0.135 & 0.113 & 0.445 \\
\hline $\mathrm{K} / \alpha$ & 0.213 & 0.860 & 0.508 & 1.000 \\
\hline $\mathrm{D}(\AA)$ & 14 & 36 & 44 & 11 \\
\hline \multicolumn{5}{|c|}{ 99:1-14.3\% MCC $2 \mathrm{PrOH}$ (2PrOH washed) } \\
\hline $\mathrm{Q}_{0}\left(\AA^{-1}\right)$ & 0.870 & 1.405 & 1.530 & 1.100 \\
\hline $2 \theta$ & 12.2 & 19.8 & 21.6 & 15.5 \\
\hline$\alpha$ & 0.470 & 0.195 & 0.108 & 0.488 \\
\hline $\mathrm{K} / \alpha$ & 0.164 & 1.198 & 0.124 & 1.000 \\
\hline $\mathrm{D}(\AA)$ & 10 & 25 & 46 & 10 \\
\hline \multicolumn{5}{|c|}{$50: 50-14.3 \% \mathrm{MCC} 2 \mathrm{PrOH}$ (water-washed) } \\
\hline $\mathrm{Q}_{0}\left(\AA^{-1}\right)$ & 0.870 & 1.410 & 1.530 & 1.120 \\
\hline
\end{tabular}


Table 2 continued

\begin{tabular}{lllll}
\hline Crystalline peak $(2 \theta)$ & $2 \theta \approx 12^{\circ}(1 \overline{1} 0)$ & $2 \theta \approx 20^{\circ}(110)$ & $2 \theta \approx 22^{\circ}(020)$ & Amorphous peak \\
\hline $2 \theta$ & 12.2 & 19.9 & 21.6 & 15.8 \\
$\alpha$ & 0.300 & 0.150 & 0.110 & 0.450 \\
$\mathrm{~K} / \alpha$ & 0.167 & 0.812 & 0.439 & 1.000 \\
$\mathrm{D}(\AA)$ & 16 & 33 & 45 & 11 \\
$50: 50-14.3 \% \mathrm{MCC} 2$ PrOH $(2 \mathrm{PrOH}$ washed $)$ & & & \\
$\mathrm{Q}_{0}\left(\AA^{-1}\right)$ & 0.870 & 1.405 & 1.530 & 1.100 \\
$2 \theta$ & 12.2 & 19.8 & 21.6 & 15.5 \\
$\alpha$ & 0.500 & 0.185 & 0.088 & 0.508 \\
$\mathrm{~K} / \alpha$ & 1.083 & 0.115 & 1.000 \\
$\mathrm{D}(\AA)$ & 0.092 & 27 & 56 & 10 \\
\hline
\end{tabular}

The peak heights $(\mathrm{K} / \alpha)$ have been normalized to the coefficients of the amorphous peak. Because the $(020)$ peak overlaps with the (110) peak, only the (110) peak width is generally of any significant precision. For samples coagulated in water, the (1 $\overline{1} 0)$ peak can also be significant relative to the amorphous scattering and can provide information about crystallite size

moderate increase with increased cellulose concentration. This dependence is more pronounced when coagulation is performed in water than when $2 \mathrm{PrOH}$ is used. Surprisingly, coagulation in $2 \mathrm{PrOH}$ and washing in water gives the largest dependence of SSA on cellulose concentration.

\section{XRD}

The WAXS spectra with deconvolutions for a selection of samples are shown in Fig. 5. The three crystalline peaks of cellulose II at $\left[\begin{array}{lll}0.87 & 1.41 & 1.53\end{array}\right]$ $\AA^{-1}$ are distinguishable from the amorphous scattering to variable degrees in Fig. 5. The two peaks of (110) and (020), at 1.41 and $1.53 \AA^{-1}$, respectively, are very close. This fact tends to obscure the $(020)$ peak at $1.53 \AA^{-1}$, which has previously been found at $1.56 \AA^{-1}\left(2 \theta=22^{\circ}\right)$ (French and Cintrón 2013). It was not possible to fit a peak at $1.56 \AA^{-1}$ to our data, but there is no explanation for the inconsistency with previous research. The other peaks are consistent with previous investigations. The peak at $1.53 \AA^{-1}$ cannot be found in any other cellulose polymorph spectrum either. The parameters, $q_{0}, \alpha, K / \alpha, 2 \theta$, and the evaluated crystallite thickness, D, from the deconvolutions in Fig. 5 are summarized in Table 2. All of the spectra of samples coagulated in $2 \mathrm{PrOH}$ and then washed in $2 \mathrm{PrOH}$ were identical. The spectra changed slightly when the material was washed in water, but the spectral change caused by this washing was identical for all four different solutions. Therefore,

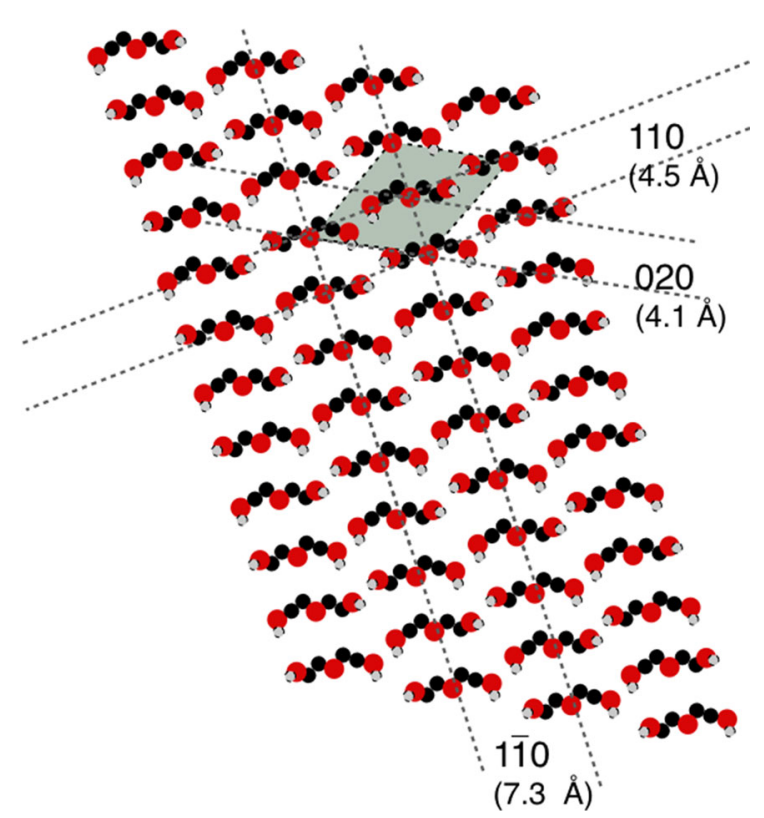

Fig. 6 The cross section of a crystallite about the size formed in water (suggested by our analysis) and the unit cell of cellulose II, grey. Note the $(020)$ reflection being at an angle between the (110) and (1 $\overline{1} 0)$ reflections (Langan et al. 2001)

only the results of 99:1-14.3\% MCC and 50:50-14.3\% MCC samples are shown in Table 2 and Fig. 5. The spectra of material coagulated in $2 \mathrm{PrOH}$ show peaks that are only barely distinguishable from the wide amorphous signal. This is an indication of very low structural order, whether crystallinity or crystallite size. Only the (110) reflection is, beyond a doubt, visible in these spectra. However, when the 
$2 \mathrm{PrOH}$-coagulated material was washed in water, the (020) reflection increased significantly. This effect was equal for all the different cellulose concentrations. Figure 5 clearly shows that even the 50:50-14.3\% MCC solutions gave spectra that only marginally differed from the 99:1-14.3\% MCC solutions when coagulated in $2 \mathrm{PrOH}$. With water as the coagulant, the structural order is much higher than with $2 \mathrm{PrOH}$ as the coagulant. With water there is also a general trend of decreasing structural order with increasing cellulose concentration or with the addition of DMSO to the solvent. Further, the process of drying from water, as opposed to performing a solvent exchange and drying from cyclohexane, clearly increases the structural order.

Before any further discussion of the results presented in Table 2, it must be stressed that only the (110) reflection yields crystallite thicknesses of any precision. Even for (110), the precision is limited to indicating the order of magnitude for the absolute dimensions of crystallites. However, significant differences $(>20 \%)$ between samples still strongly indicate trends for the (110) dimension. For coagulation in water, the $(1 \overline{1} 0)$ reflection also suggests a very rough estimation of the crystallite dimension perpendicular to the (110) plane. For example the (110)

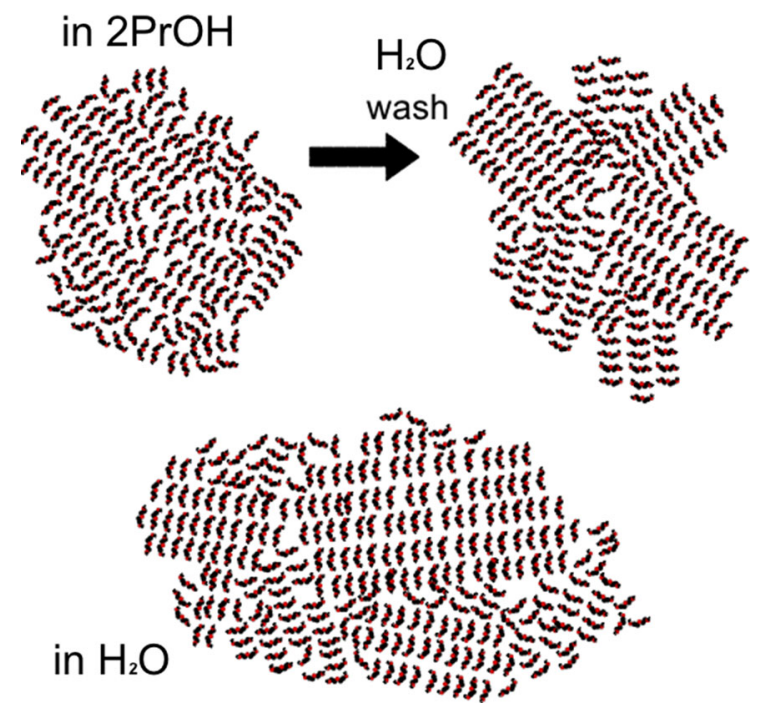

Fig. 7 Fibril cross sections made up by several crystallites of mixed sizes depending on the coagulant used, as suggested by our analysis based on Eqs. 2 and 3 (or 4). Above: rearrangement during washing in water of $2 \mathrm{PrOH}$-coagulated material. Below: larger crystallites in fibril formed in water during coagulation dimension appears to have significantly increased during regular drying from water compared to the solvent exchanged material. For $2 \mathrm{PrOH}$ coagulation, it can only be concluded that the number of planes stacked in the (110) direction is very limited, because its peak essentially disappeared in the amorphous signal. The width of the (020) peak, which is narrower than the (110) peak and, consequently, implies that crystallites are thicker in the (020) direction, is unreasonable given that the crystallites are consistently thinner in the $(1 \overline{1} 0)$ dimension. This is rather apparent considering the relative directions of the planes, cf. Figure 6, and highlights the limitations of the data due to the signal overlap and the unknown shape of the amorphous scattering. It must also be clarified that the dimensions given in Table 2 represent the upper fraction out of a wide distribution of crystallite dimensions. The scattering from smaller ordered domains could not be distinguished from the amorphous scattering and the larger crystallites. Thus it cannot be enough emphasized that the dimensions deduced from the pwhm and discussed below are the dimensions suggested by the Scherrer equation (Eq. 2) and only for the crystallites that are large enough to give significant signals. The precision is limited to the first digit, at best, and deteriorates as dimensions approach the dimensions of the unit cell.

The largest crystallites were found in the material coagulated in water from the $99: 1-5 \% \mathrm{MCC}$ solution and the analysis suggests a size of about 7 by $3 \mathrm{~nm}$, or about 15 planes stacked in the (110) direction and 4-5 planes in the (110) direction. In the other solutions coagulated in water, the (110) dimension was smaller, close to $5 \mathrm{~nm}$. Our analysis suggests that crystallite cross sections as large as that in Fig. 6 are produced during coagulation in water. Coagulation in $2 \mathrm{PrOH}$ generates smaller crystallites; based on our measurements and analysis, less than $\sim 3 \mathrm{~nm}$ is generated in the (110) direction and $\sim 1 \mathrm{~nm}$ in the (110) direction, i.e. just one or two (110) planes. It can be discussed whether these should be termed crystallites. However, the sudden appearance of the (020) reflection, upon washing in water, indicates that such "all surface" crystallites, which show only the (110) reflection, did stack up on their (11̄0) planes, when exposed to water, as shown in the upper part of Fig. 7. Since the (020) plane is at an angle between the (110) and (110) planes, it was visible. The (110) reflection, however, 
probably increased but from too low levels to be visible in the spectra. The relative widths and amplitudes of the (110) and (1 $\overline{10})$ peaks indicate that, with both non-solvents but particularly with $2 \mathrm{PrOH}$, cellulose has a tendency to selectively stack up on its hydrophobic planes. This has previously been observed in aqueous systems, alkaline solutions, where it was more easily explained as a consequence of the hydrophobic effect (Yamane et al. 2015). In the present case of an IL with minor amounts of nonsolvent it is more reasonable to explain the selective stacking of hydrophobic (110) planes by IL anions blocking the stacking on its hydrophilic side. With the $\mathrm{OH}$ groups blocked, the only alternative for cellulosecellulose interaction is through the hydrophobic plane. This would also be consistent with the larger selectivity for hydrophobic stacking in $2 \mathrm{PrOH}$ coagulation due to its lower ability to remove IL anions from the cellulose. This will be discussed in the next subsection.

The fraction of amorphous scattering in the deconvoluted spectra in Fig. 5 varies significantly among samples, but its fraction is also somewhat dependent on where it is centered, as it substitutes for the (110) peak if the amorphous scattering and the (110) peak overlap. "Amorphous cellulose" is not the definition of a certain well-defined structure, but a term that can be applied to any cellulose material in which the molecules are not well organized. The disorderly organization of the molecules can be subject to variations due to process conditions. Consequently, the exact curve shape from amorphous scattering is specific for each type of material. In our data, and using the function $L(q) * q$ to model it, see the Materials and methods section, the amorphous scattering was centered at lower $q$ values in the spectra of less-ordered material (coagulated in $2 \mathrm{PrOH}$ ), than in the spectra of more-ordered material (coagulated in water). Thus, it is reasonable to consider what such amorphous material might be composed of and what might be the cause of such variation. If attempting to produce deconvolutions based only on crystalline peaks at $\left[\begin{array}{lll}0.87 & 1.41 & 1.53\end{array}\right] \AA^{-1}$, but of infinitely many widths, it becomes apparent that the amorphous scattering contains a bulge around $1.2 \AA^{-1}$ that can never be emulated without a peak that is centered close to the bulge. Yet, a significant part of the "amorphous" scattering may very well originate from crystallites that are much thinner than the dimensions evaluated using deconvolutions and summarized in Table 2. Those larger crystallites are probably just some upper percentile of an actual distribution of crystallite sizes. The relative dimensions of such finer crystallites, i.e. the peak widths of the (1 $1 \overline{1} 0)$ reflection in the low range and the (110) and (020) reflections in the high range, could be one factor behind the shifting of the amorphous peak center. If the crystallites are thinner perpendicularly to one of these planes, the contribution to the "amorphous" scattering around that reflection from small crystallites will be greater. The bulge around $1.2 \AA^{-1}$ could be due to both intercrystalline interfaces (grain boundaries) and local defects. In particular, the scattering generated by the interfaces is likely dependent on the shapes and dimensions of the crystallites they connect. This could also cause the amorphous peak to shift. It appears unlikely that a general model for amorphous cellulose can be found, e.g. the spectrum of ball-milled cellulose. At least, it is not necessarily better than any other random function that fits the data to be analyzed. A model spectrum for amorphous scattering must be withdrawn from WAXS spectra when determining the crystalline index (CI) or peak widths. The CI of coagulated material is often calculated based on the ratio of crystalline peak integrals to the whole signal integral, which also includes the amorphous parts (Krässig 1993). However, this approach requires that the peak widths are constant for all samples, which is not consistent with the theory of line broadening on which, e.g. the Scherrer equation relies. If evaluating CIs based on the peak deconvolutions of this data, spectra that are obviously dominated by amorphous material can yield CIs similar to apparently more crystalline spectra. This problem with reducing spectra into scalar CI values is caused by the interchangeability between very wide crystalline peaks and amorphous scattering. A wide crystalline peak has a large area relative to its height, and this large area is not easily distinguishable from the amorphous scattering.

\section{Fibril cross sections}

Below, the constitution of a fibril cross section will be discussed, based on the lateral dimensions of crystallites and fibrils that were indicated by the analysis of 
$\mathrm{XRD}$ - and BET data respectively. The roughness, in particular of XRD data deconvolutions, limits the precision of structural insight. The analysis suggests general structural features and some relative trends of interest, given certain assumptions, i.e. not exact quantitative data. The fibril cross sections are likely to be bundles constituted of a small number $(\sim 5-30)$ of crystallite cross sections, possibly something like the simplified structure depicted in Fig. 7. Fibrils less than $10 \mathrm{~nm}$ in diameter can only contain, at the most, a couple of crystallites that are 5-7 $\mathrm{nm}$ wide. However, crystallite sizes vary, and most crystallites inside a fibril are smaller. The size distribution of those smaller crystallites is obscured because their scattering is indistinguishable from amorphous scattering. The general features are qualitatively similar to the previous investigations of never dried NMMO fibers spun in water (Fink et al. 2001).

An increase in cellulose concentrations reduced the thicknesses of both fibrils and crystallites formed during coagulation in water and increased the amorphous scattering relative to the crystalline peaks, cf. Table 2. Consequently, in water, there is a strong correlation between a high cellulose concentration and a low crystalline order. The data indicates that the effects of cellulose concentration are more pronounced for crystallite thickness than for fibril thickness. Thus, probably, there are more small crystallites per fibril in the material from the $25 \mathrm{wt} \%$ solution than from the $5 \mathrm{wt} \%$ solution. The solutions with more DMSO (50:50-14.3 wt\%MCC) generated the largest number of small crystallites per fibril, for coagulation in water, based on its significantly lower crystalline order. The very low degree of crystalline order (many thin crystallites) and the slightly lower SSAs (thicker fibrils) found for coagulation in $2 \mathrm{PrOH}$, imply that the number of crystallites per fibril should be significantly larger for coagulation in $2 \mathrm{PrOH}$ than in water.

The selectivity for hydrophobic stacking and crystallite growth perpendicular to the (110) plane that was observed, could cause the fibril cross sections as well to become elongated in the (110) direction, as exemplified in the lower part of Fig. 7. Our data or SEM images cannot disclose whether this is the case. However, if it were very pronounced and made the fibrils resemble ribbons rather than fibrils, Eq. 4 would have to replace Eq. 3 for the calculation of fibril thickness. $h=2 /(\rho * S S A)$

The thickness $h$ of such a "nano ribbon" would be only $4-5 \mathrm{~nm}$ and could not allow more than $\sim 2$ crystallites to be stacked on their larger flat side for coagulation in water. For $2 \mathrm{PrOH}$, our analysis sug-

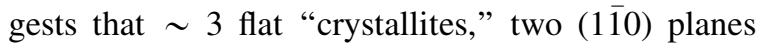
thick each, could be stacked to produce the thickness of such "nano ribbons." This implies that the smallest fibril dimension would be slightly smaller if the fibrils are ribbons, but the general picture remains largely unaffected by the shape of the cross section.

Mechanistic explanations

Traditional approaches to explaining the differences in the crystalline order, porosity and SSA of regenerated material between water and $2 \mathrm{PrOH}$ coagulation would involve differences in surface tension between water and $2 \mathrm{PrOH}$. The larger surface tension of water could act to organize cellulose chains into crystalline domains by hydrophobic stacking, which $2 \mathrm{PrOH}$ would not, as previously observed in aqueous $\mathrm{NaOH}$ cellulose solutions (Yamane et al. 2015). The larger fibrils and pores generated by $2 \mathrm{PrOH}$ could similarly be the result of greater liquid-liquid surface tension of the interface between an $\left[\mathrm{C}_{2} \mathrm{mim}\right][\mathrm{OAc}]$-cellulose phase and a non-solvent rich phase. An $\left[\mathrm{C}_{2-}\right.$ mim][OAc]-cellulose phase in contact with $2 \mathrm{PrOH}$ should imply greater surface tension than in contact with water, for which both $\left[\mathrm{C}_{2} \mathrm{mim}\right][\mathrm{OAc}]$ and cellulose have a much higher affinity. However, unlike the aqueous $\mathrm{NaOH}$ solutions studied by Yamane et al., $\left[\mathrm{C}_{2} \mathrm{mim}\right][\mathrm{OAc}]$ solutions are quite far from being dominated by water or $2 \mathrm{PrOH}$ at the time of coagulation and crystallization, as only about 5-35 wt $\%$ of coagulant is required to cause phase separation (water: 5-25 and 2PrOH: 8-35 wt\%). (Hedlund et al. 2015) Consequently, a hydrophobic effect from 5 and $14 \mathrm{wt} \%$ water in the 99:1-25\% MCC and 99:1-14.3\%MCC solutions, respectively, seems an unlikely explanation for the higher degree of crystalline order found in material coagulated in water than in $2 \mathrm{PrOH}$.

In another study, in which the results are somewhat similar to this study, the increasing porosities and the decreasing crystalline order and orientation of NMMO fibers spun into alcohols of increasing molecular weight was attributed to soft (slow) and hard (fast) 

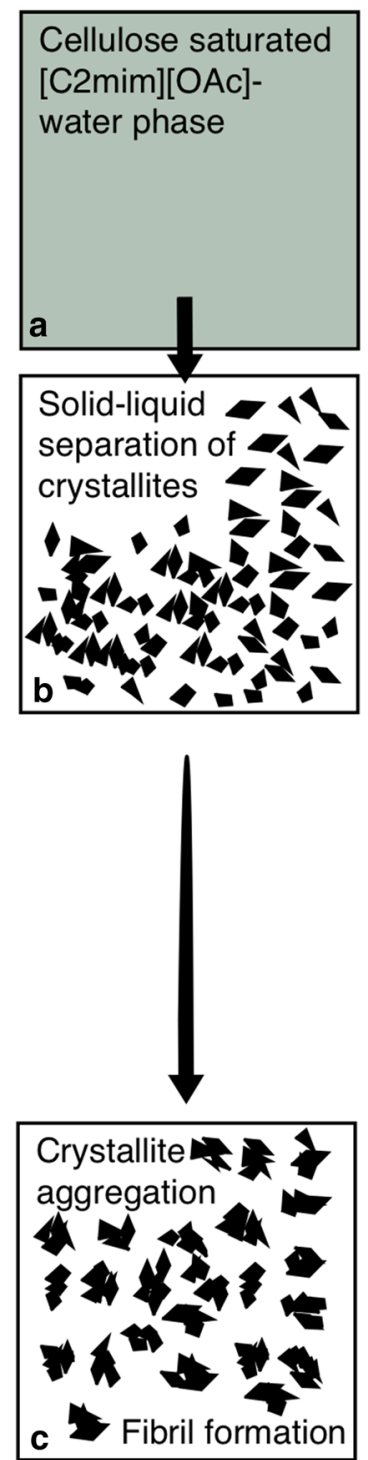

Fig. 8 Schematic 2D cross sections of a volume undergoing phase separation and fibril formation by the different routes hypothesized. Water coagulation a-c: a single-phase mixture of water, $\left[\mathrm{C}_{2} \mathrm{mim}\right][\mathrm{OAc}]$ and cellulose; b solid-liquid phase separation into solid cellulose crystallites (cross sections, black) and liquid $\left[\mathrm{C}_{2} \mathrm{mim}\right][\mathrm{OAc}]-$ water phase (white); c crystallites aggregated into fibrils. $2 \mathrm{PrOH}$ coagulation $\mathrm{d}-\mathrm{g}$ : $\mathbf{d}$ single-phase $2 \mathrm{PrOH},\left[\mathrm{C}_{2} \mathrm{mim}\right][\mathrm{OAc}]$ and cellulose mixture; e liquid-liquidphase separation into major cellulose-[ $\left.\mathrm{C}_{2} \mathrm{mim}\right][\mathrm{OAc}]$ liquid phase (grey) and minor liquid $2 \mathrm{PrOH}-\left[\mathrm{C}_{2} \mathrm{mim}\right][\mathrm{OAc}]$ phase droplets; $\mathbf{f}$ gradual cellulose concentration increase as $\left[\mathrm{C}_{2-}\right.$ $\operatorname{mim}][\mathrm{OAc}]$ diffuses out; $\mathrm{g}$ secondary phase separation of continuous phase into bicontinuous fibril network and $\left[\mathrm{C}_{2}\right.$ $\operatorname{mim}][\mathrm{OAc}]-2 \mathrm{PrOH}$ phase coagulation, based on the slower diffusion of larger alcohols. (Fink et al. 2001) The conception of hard versus soft coagulation emphasizes coagulation time, which might be more valid for oriented structures and in particular orientation that relaxes with time. Porosity could be attributed to a longer time during which pores can Ostwald-ripen or grow by aggregation, but a negative effect of slow coagulation on crystallinity is not evident. Molecular mobility is a parameter that typically correlates to crystalline order and domain sizes in polymer materials. For a comparison of water and $2 \mathrm{PrOH}$, it is not obvious which coagulant would enhance the mobility of cellulose in solution the most: $2 \mathrm{PrOH}$ by means of the larger amounts (by weight) required to induce coagulation, or water due to its smaller size and the larger number of molecules required to induce coagulation. However, the effect of DMSO on reducing crystalline order in water coagulation and the lack of cellulose-concentration dependence in $2 \mathrm{PrOH}$ coagulation are two examples of complexities that cannot be explained by the bulk parameters of mobility, such as viscosity or diffusivity, or by coagulation rate.

An alternative explanation begins with the assumption of different types of phase separations for water and $2 \mathrm{PrOH}$. This would be mainly due to fundamental differences between their interactions with cellulose. Water interacts readily with both $\left[\mathrm{C}_{2} \mathrm{mim}\right][\mathrm{OAc}]$ and cellulose because of its polarity and ability to both donate and accept hydrogen bonds, whereas $2 \mathrm{PrOH}$, although still miscible with $\left[\mathrm{C}_{2} \mathrm{mim}\right][\mathrm{OAc}]$, has much weaker interactions with the other two components of the mixture. In other words, it is likely that water, when used as the coagulant, mixes evenly throughout the precipitating solution (Fig. 8a) until solid cellulose crystallites separate from the remaining mixture of $\left[\mathrm{C}_{2} \mathrm{mim}\right][\mathrm{OAc}]$ and water as in Fig. $8 \mathrm{~b}$, which shows small polygons that represent cross sections of the long slender crystallites formed. This study has assumed that crystallites must be thin and elongated because of the stiffness of cellulose molecules. This means that it is very favorable for the cellulose molecules to be oriented in parallel relative to crossing each other at a significant angle. These slender crystallites aggregate into fibrils either directly or later during further removal of solvent from the surrounding non-solvent phase, as in Fig. 8c. Under these conditions, cellulose molecules aggregate because of mutual attraction rather than exclusion by 
the solvent-non-solvent mixture. The mixture probably still interacts very favorably with the mainly hydrophilic crystallite surfaces, cf. Figure 6 or the dominant (110) peak. Such conditions could stabilize even very small crystallites.

Based on the phase diagram, solutions with less cellulose will also have more water present at the time of phase separation (Hedlund et al. 2015). Both of these factors should increase mobility and favor crystalline order in the coagulating solution. This would explain the correlation between crystalline order and cellulose concentration observed during coagulation in water. It is plausible that water affects the local mobility of IL-anions in close proximity of cellulose molecules and, consequently, also the mobility of cellulose. It has been shown (Bengtsson et al. 2017) through molecular dynamics simulations that water is somewhat concentrated to the proximity of, and interacts with, the cellulose chains, when present in IL-cellulose solutions. While reducing ILcellulose interactions and reducing solvent quality, due to its ability to donate hydrogen bonds, water also accepts hydrogen bonds from cellulose to act, in a certain sense, as a solvent for cellulose. This duality of water likely speeds up the shifting of hydrogen bonds between different molecules and hydroxyl groups by supplying intermediate bonds to reduce the energy barrier of hydrogen bond movement and thus to increase the frequency of hydrogen bond rearrangements. That study did not find the same for an alcohol. The increased mobility and faster crystallization in water could also favor solid-liquid over liquid-liquid phase separation and, thus, influence the mode of separation. The decrease in fibril size with increasing cellulose concentration for samples coagulated in water is probably an effect of lower crystallite mobility in materials with more crystallites per volume, during the secondary aggregation of crystallites into fibrils.

We suggest that also $2 \mathrm{PrOH}$ would initially spread evenly, as in Fig. 8e, up to a concentration at which the ternary solubility of $2 \mathrm{PrOH},\left[\mathrm{C}_{2} \mathrm{mim}\right][\mathrm{OAc}]$ and cellulose is limited. However, at that concentration, a liquid-liquid phase separation would be induced, as in Fig. 8e. Then a liquid $2 \mathrm{PrOH}-\left[\mathrm{C}_{2} \mathrm{mim}\right][\mathrm{OAc}]$ phase and a very viscous but yet liquid $\left[\mathrm{C}_{2} \mathrm{mim}\right][\mathrm{OAc}]-$ cellulose phase would result. The $\left[\mathrm{C}_{2} \mathrm{mim}\right][\mathrm{OAc}]$ solvent would be divided between the cellulose- and $2 \mathrm{PrOH}-$ rich phases. If the cellulose concentration is high, cellulose will form the major continuous phase with isolated drops of non-solvent rich phase, as in e. Given a certain cellulose concentration, the relative volume ratio of the two liquid phases depends on the relative attractive forces between the species and on the concentration of the non-solvent. As more $2 \mathrm{PrOH}$ diffuses into and $\left[\mathrm{C}_{2} \mathrm{mim}\right][\mathrm{OAc}]$ diffuses out of the coagulating solution, the $2 \mathrm{PrOH}$ phase increases in terms of volume fraction, which leads to a gradual and relatively slow depletion of $\left[\mathrm{C}_{2} \mathrm{mim}\right][\mathrm{OAc}]$ from the shrinking cellulose-rich phase, as in Fig. $8 \mathrm{f}$. In the cellulose rich phase, $\left[\mathrm{C}_{2} \mathrm{mim}\right][\mathrm{OAc}]$ sterically inhibits crystallization due to the anions' attachment by hydrogen bonds to cellulose hydroxyl groups at random locations along the chain. Due to the relatively low affinity of alcohols for cellulose, there will be no enrichment of non-solvent molecules in close proximity to the chain, but rather the opposite. Thus, alcohols will not increase the mobility of ions and molecules in proximity to cellulose chains, as we have hypothesized to be the case with water. The actual solidification and possibility to crystallize would not occur until the critical solubility of cellulose in $\left[\mathrm{C}_{2} \mathrm{mim}\right][\mathrm{OAc}]$ (with some minor $2 \mathrm{PrOH}$ ) has been surpassed, i.e. above $\sim 30 \mathrm{wt} \%$, as in Fig. $8 \mathrm{~g}$. The major domain in Fig. 8 (f) goes through a secondary phase separation in which solid fibrils are formed as in Fig. 8g. The key point of the proposed explanation is that the secondary separation begins with a highly concentrated cellulose-rich phase. The molecular mobility at room temperature in such a phase is very limited and, consequently, prohibits any significant organization of macromolecules into large crystallites. Due to the primary liquid-liquid phase separation and the gradual $\left[\mathrm{C}_{2} \mathrm{mim}\right][\mathrm{OAc}]$ depletion of the cellulose rich phase, all solutions will, regardless of their initial cellulose concentration, solidify from about the same very high local cellulose concentration in the liquid cellulose-rich phase. The only difference between solutions of different cellulose concentration is the volume fraction of cellulose rich phase. This would explain the low crystalline order and its surprising independence of the cellulose concentration in solutions coagulated in $2 \mathrm{PrOH}$.

Also the fact that the variation in fibrillar size is very limited over a wide range of cellulose concentrations can be explained by liquid-liquid phase separation that is followed by a secondary phase separation in the cellulose-rich liquid phase. If the 
concentrations in a cellulose-rich liquid phase at the time of fibril formation are the same for all initial cellulose concentrations, the surface tension between the liquid phases and most other possibly important conditions would also be the same, which would give equal dimensions for the final fibrils. The large pores formed during coagulation in $2 \mathrm{PrOH}$ and observed with SEM are also consistent with the hypothesis that liquid-liquid is the primary mode of separation.

Finally, the unexpected decrease in the degree of crystalline order in material coagulated in water caused by an increase in DMSO content can be explained similar to the effects of $2 \mathrm{PrOH}$ as a coagulant. The interaction of DMSO with water is much more enthalpically favorable than DMSOcellulose interaction. The acetate anion prefers water, but it readily accepts hydrogen bonds from either water or cellulose. The result is a local relative increase in acetate-ion concentration and a local relative decrease in the concentrations of DMSO and water around the cellulose chains. DMSO and water are instead concentrated at some distance from the cellulose chain, due to their strong tendency to interact with each other. The local mobility around cellulose chains is reduced as a result of the lower water to IL ratio and lower frequency with which hydrogen bonds are broken and created. Consequently, the crystalline order of the 50:50-14.3\% MCC sample was reduced relative to the $99: 1-14.3 \% \mathrm{MCC}$ sample coagulated in water. However, it was still significantly higher than the corresponding samples coagulated in $2 \mathrm{PrOH}$. The greater abundance of larger pores in the 50:50-14.3\%MCC material, observed in the SEM pictures, suggests that liquid-phase drops had been formed. Thus, it appears that the primary mode of coagulation may have changed from solid-liquid to liquid-liquid, which would reduce the crystalline order.

The labile state of $2 \mathrm{PrOH}$ coagulated material is exhibited by the radical structural changes induced by washing in water. The SSA of such material may either increase or decrease from washing in water, depending on the cellulose concentration, whereas the crystalline order increases for all cellulose concentrations. It was found that the $2 \mathrm{PrOH}-$ coagulated cellulose material shrinks when washed in water. The fundamentality of this restructuring is underlined by the width of scales, from nano- to the macroscale, at which properties change. It is of significance that the restructuring is not from drying, as in the much discussed hornification processes, but merely from the swelling in water. This is probably a consequence of the combination of the cellulose-swelling ability of water and the very low degree of order before swelling, which makes the cellulose swell more than usual. The restructuring during swelling might be similar to that depicted in Fig. 8. Some degree of fibril shrinkage to reduce surface energies would also be expected as mobility is reintroduced into a labile structure that has not been allowed to relax into a thermodynamically favorable state. The increase in SSA of the samples from the 14.3 and $25 \mathrm{wt} \%$ solutions after washing in water is contrary to the general assumption that structures reformed after swelling and reorganization are thermodynamically more favorable. An explanation could be that more free energy is lost through crystallization than is gained to create the larger surfaces when the crystallites are poorly organized into fibrils, as in Fig. 8.

\section{Conclusion}

Materials coagulated from $\left[\mathrm{C}_{2} \mathrm{mim}\right][\mathrm{OAc}]$-cellulose solutions in water or alcohols were found to be distinctly different, particularly in terms of structural order. The crystalline order and crystallite size were reduced if coagulation was performed in $2 \mathrm{PrOH}$ instead of water. Although both types of coagulants generated fibrils of similar diameters connected in open-pore networks, the heterogeneity and size of the pores were larger if the cellulose concentrations were lower or if alcohols replaced water as the coagulant. These observations were explained in terms of different modes of phase separation, i.e. the occurrence of liquid-liquid- or liquid-solid phase separation, which in turn is determined by the relative affinities between the various components involved.

Acknowledgments This research could not have been without the financing from the Södra Skogsägarna Foundation for Research, Development and Education. The partners within the Avancell project have provided inspiring discussions and feedback. Our helpful colleague and SEM expert at Swerea IVF, Melina da Silva, was also essential to these results.

Open Access This article is distributed under the terms of the Creative Commons Attribution 4.0 International License (http:// creativecommons.org/licenses/by/4.0/), which permits unrestricted use, distribution, and reproduction in any medium, 
provided you give appropriate credit to the original author(s) and the source, provide a link to the Creative Commons license, and indicate if changes were made.

\section{References}

Andanson J-M, Bordes E, Devémy J, Leroux F, Pádua AA, Gomes MFC (2014) Understanding the role of co-solvents in the dissolution of cellulose in ionic liquids. Green Chem 16:2528-2538

Balta-Calleja F, Vonk C (1989) X-ray scattering of synthetic polymers, vol 8. Elsevier Science Ltd, Amsterdam

Bang YH, Lee S, Park JB, Cho HH (1999) Effect of coagulation conditions on fine structure of regenerated cellulosic films made from cellulose/ $\mathrm{N}$-methylmorpholine- $\mathrm{N}$-oxide/H2O systems. J Appl Polym Sci 73:2681-2690

Bengtsson J, Olsson C, Hedlund A, Köhnke T, Bialik E (2017) Understanding the inhibiting effect of small-molecule hydrogen bond donors on the solubility of cellulose in tetrabutylammonium acetate/DMSO. J Phys Chem B 121:11241-11248

Brunauer S, Emmett PH, Teller E (1938) Adsorption of gases in multimolecular layers. J Am Chem Soc 60:309-319

Burghardt W, Yilmaz L, McHugh A (1987) Glass transition, crystallization and thermoreversible gelation in ternary PPO solutions; relationship to asymmetric membrane formation. Polymer 28:2085-2092

Collier J, Watson J, Collier B, Petrovan S (2009) Rheology of 1-butyl-3-methylimidazolium chloride cellulose solutions. II. Solution character and preparation. J Appl Polym Sci 111:1019-1027

Fink H-P, Weigel P, Purz H, Ganster J (2001) Structure formation of regenerated cellulose materials from NMMOsolutions. Prog Polym Sci 26:1473-1524

French AD (2014) Idealized powder diffraction patterns for cellulose polymorphs. Cellulose 21:885-896

French AD, Cintrón MS (2013) Cellulose polymorphy, crystallite size, and the Segal crystallinity index. Cellulose 20:583-588

Gjönnes J, Norman N, Viervoll H (1958) X-Ray Diffractograms. Acta Chem Scand 12:489-494

Gupta KM, Hu Z, Jiang J (2013a) Cellulose regeneration from a cellulose/ionic liquid mixture: the role of anti-solvents RSC. Advances 3:12794-12801

Gupta KM, Hu Z, Jiang J (2013b) Molecular insight into cellulose regeneration from a cellulose/ionic liquid mixture: effects of water concentration and temperature. RSC Adv 3:4425-4433

Härdelin L, Thunberg J, Perzon E, Westman G, Walkenström P, Gatenholm P (2012) Electrospinning of cellulose nanofibers from ionic liquids: the effect of different cosolvents. J Appl Polym Sci 125:1901-1909

Hauru LK, Hummel M, King AW, Kilpeläinen I, Sixta H (2012) Role of solvent parameters in the regeneration of cellulose from ionic liquid solutions. Biomacromol 13:2896-2905

Hedlund A, Köhnke T, Theliander H (2015) Coagulation of EmimAc-cellulose solutions: dissolution-precipitation disparity and effects of non-solvents and cosolvent. Nord Pulp Pap Res J 30:32-42

Hedlund A, Köhnke T, Theliander H (2017) Diffusion in ionic liquid-cellulose solutions during coagulation in water: mass transport and coagulation rate measurements. Macromolecules 50:8707-8719. https://doi.org/10.1021/ acs.macromol.7b01594

Hermanutz F, Gähr F, Uerdingen E, Meister F, Kosan B (2008) New developments in dissolving and processing of cellulose in ionic liquids. In: Macromolecular symposia. Wiley Online Library, pp 23-27

Idström A et al (2017) On the dissolution of cellulose in tetrabutylammonium acetate/dimethyl sulfoxide: a frustrated solvent. Cellulose 24:3645-3657

Kosan B, Michels C, Meister F (2008) Dissolution and forming of cellulose with ionic liquids. Cellulose 15:59-66

Kosan B, Schwikal K, Meister F (2010) Solution states of cellulose in selected direct dissolution agents. Cellulose 17:495-506

Krässig HA (1993) Cellulose: structure, accessibility and reactivity. Gordon and Breach Science Publishers, London

Langan P, Nishiyama Y, Chanzy H (2001) X-ray structure of mercerized cellulose II at $1 \AA$ resolution. Biomacromol $2: 410-416$

Le KA, Sescousse R, Budtova T (2012) Influence of water on cellulose-EMIMAc solution properties: a viscometric study. Cellulose 19:45-54

Le KA, Rudaz C, Budtova T (2014) Phase diagram, solubility limit and hydrodynamic properties of cellulose in binary solvents with ionic liquid. Carbohydr Polym 105:237-243

Lu F, Wang L, Zhang C, Cheng B, Liu R, Huang Y (2015) Influence of temperature on the solution rheology of cellulose in 1-ethyl-3-methylimidazolium chloride/dimethyl sulfoxide. Cellulose 22:3077-3087

Michud A, Hummel M, Sixta H (2015) Influence of molar mass distribution on the final properties of fibers regenerated from cellulose dissolved in ionic liquid by dry-jet wet spinning. Polymer 75:1-9

Michud A et al (2016) Ioncell-F: ionic liquid-based cellulosic textile fibers as an alternative to viscose and lyocell. Text Res J 86:543-552

Olsson C, Westman G (2013) Wet spinning of cellulose from ionic liquid solutions: viscometry and mechanical performance. J Appl Polym Sci 127:4542-4548

Olsson C, Hedlund A, Idström A, Westman G (2014) Effect of methylimidazole on cellulose/ionic liquid solutions and regenerated material therefrom. J Mater Sci 49:3423-3433

Parviainen A et al (2013) Predicting cellulose solvating capabilities of acid-base conjugate ionic liquids. Chemsuschem 6:2161-2169. https://doi.org/10.1002/cssc.201300143

Radhi A, Le KA, Ries ME, Budtova T (2015) Macroscopic and microscopic study of 1-ethyl-3-methyl-imidazolium acetate-DMSO mixtures. J Phys Chem B 119:1633-1640

Saba H, Zhu X, Chen Y, Zhang Y (2014) Determination of physical properties for the mixtures of [BMIM] $\mathrm{Cl}$ with different organic solvents. Chin J Chem Eng 23:804-811

Sammons R, Collier J, Rials T, Petrovan S (2008a) Rheology of 1-butyl-3-methylimidazolium chloride cellulose solutions. I. Shear rheology. J Appl Polym Sci 110:1175-1181

Sammons RJ, Collier JR, Rials TG, Petrovan S (2008b) Rheology of 1-butyl-3-methylimidazolium chloride cellulose 
solutions. III. Elongational rheology. J Appl Polym Sci 110:3203-3208

Song H, Niu Y, Wang Z, Zhang J (2011) Liquid crystalline phase and gel-sol transitions for concentrated microcrystalline cellulose (MCC)/1-ethyl-3-methylimidazolium acetate (EMIMAc) solutions. Biomacromol 12:1087-1096

Swatloski RP, Spear SK, Holbrey JD, Rogers RD (2002) Dissolution of cellose with ionic liquids. J Am Chem Soc 124:4974-4975

The_Fiber_Year_GmbH (2017) The Fiber Year (2017) World survey on textiles \& nonwovens, vol 16. Speicher, Switzerland

Van de Witte P, Dijkstra P, Van den Berg J, Feijen J (1996) Phase separation processes in polymer solutions in relation to membrane formation. J Membr Sci 117:1-31

Wang S, Lu A, Zhang L (2016) Recent advances in regenerated cellulose materials. Prog Polym Sci 53:169-206
Wawro D, Hummel M, Michud A, Sixta H (2014) Strong cellulosic film cast from ionic liquid solutions. In: Fibres and Textiles in Eastern Europe

Xu S, Zhang J, He A, Li J, Zhang H, Han CC (2008) Electrospinning of native cellulose from nonvolatile solvent system. Polymer 49:2911-2917

Yamane C, Hirase R, Miyamoto H, Kuwamoto S, Yuguchi Y (2015) Mechanism of structure formation and dissolution of regenerated cellulose from cellulose/aqueous sodium hydroxide solution and formation of molecular sheets deduced from the mechanism. Cellulose 22:2971-2982

Zhang J, Zhang H, Wu J, Zhang J, He J, Xiang J (2010) NMR spectroscopic studies of cellobiose solvation in EmimAc aimed to understand the dissolution mechanism of cellulose in ionic liquids. Phys Chem Chem Phys 12:1941-1947 